\title{
Spontaneous Low-Frequency Fluctuation Observed with Functional Magnetic Resonance Imaging as a Potential Biomarker in Neuropsychiatric Disorders
}

\author{
Yuan Zhou ${ }^{1,2}$, Kun Wang ${ }^{2}$, Yong Liu², Ming Song², \\ Sonya W Song', and Tianzi Jiang ${ }^{2}$, CA \\ ${ }^{1}$ Center for Social and Economic Behavior, Institute of Psychology, Chinese Academy of \\ Sciences, Beijing, PR. China \\ ${ }^{2}$ LIAMA Center for Computational Medicine, National Laboratory of Pattern \\ Recognition, Institute of Automation, Chinese Academy of Sciences, Beijing, PR. China
}

\{Tianzi Jiang, jiangtz\}@nlpr.ia.ac.cn

\begin{abstract}
As functional magnetic resonance imaging (fMRI) studies have yielded increasing amounts of information about the brain's spontaneous activity, they have revealed fMRI's potential to locate changes in brain hemodynamics in neuropsychiatric disorders. In this paper, we review studies that support the notion that changes in spontaneous low-frequency fluctuation (SLFF) observed by fMRI can be used as potential biomarkers for diagnosis and treatment evaluation in neuropsychiatric disorders. In this paper, we review the methods used to study SLFF from individual region of interest analysis, to local network analysis, to whole brain network analysis. We also summarize the major findings associated with major neurological and psychiatric disorders obtained using these methods.
\end{abstract}

Keywords: Resting-state fMRI; Low frequency fluctuation; Functional connectivity; ICA; Alzheimer's disease; Schizophrenia

\section{Introduction}

Accurate diagnosis of neuropsychiatric disorders presents a major challenge for psychiatrists and clinicians. The current situation, in which neuropsychiatric disorders are diagnosed mainly based on clinical symptoms and medical history, has spurred the search for objective biomarkers, such as neuroimaging markers.

Spontaneous brain activity, as observed by functional magnetic resonance imaging (fMRI), is termed spontaneous low-frequency fluctuation (SLFF) in blood oxygen level dependence (BOLD) signal and has recently attracted the attention of researchers as a potential biomarker for locating changes in brain hemodynamics associated with diseases because of its clinical advantages, such as non-invasive, easily performed [1, 2]. Findings of the physiological origin of spontaneous brain 
activity further strengthen the possibility that detected changes in spontaneous brain activity can be used as potential biomarkers (for reviews, see [1, 3]). This has inspired more and more researchers to explore whether endogenous fMRI markers can characterize the neurophysiological changes associated with disease or drug treatments. To date many studies have been performed involving nearly all of the major neurological and psychiatric disorders. Various methods for detecting spontaneous activity have been used to investigate biomarkers that can be related to specific neuropsychiatric disorders. In this paper, we review the methods used to study SLFF from individual region of interest (ROI) analysis, to local network analysis, to whole brain network analysis. We take two representative neuropsychiatric disorders, i.e., Alzheimer's disease (AD) and schizophrenia, as examples to instantiate how the findings obtained using these methods would be helpful to find the biomarkers as diagnosis, therapy evaluation and prognosis of major neurological and psychiatric disorders.

\section{Methods and progress in understanding neuropsychiatric disorders}

Correlation or coherence of brain region activity is often thought to reflect functional integration, one of the fundamental principles of functional organization of the brain $[4,5]$. Resting-state functional connectivity (rsFC) analysis and independent component analysis (ICA) are two main methods of analysis that can be used to investigate the spatial pattern of spontaneous activity during rest.

Conventional FC measures correlations between a reference time series and another time series [6]. Using this method, fMRI studies have demonstrated that spontaneous BOLD fluctuations are coherent within specific neuro-anatomical systems, such as primary motor auditory, visual cortices, language and limbic systems in healthy subjects (for a review, please see [1]). The rsFC analysis can be divided into three categories: rsFC based on individual regions of interest (ROI), rsFC based on multiple ROIs, and rsFC based on whole brain regions.

The ICA is another commonly used method for identifying spatial patterns in resting-state fMRI data [7-11], This method, like the rsFC based on multiple ROIs, can provide specific information on local brain network. Unlike traditional FC analysis, ICA is a model-free method that decomposes the data into statistically independent components. Such decompositions are very useful because they allow for separation into different coherent resting networks and separate these networks from other effects such as head motion or other physiological confounds (such as cardiac pulsation or the respiratory cycle). The conventional ICA method can only be used for a single subject. Recently, a method for investigating coherent signals at a group level, tensor probabilistic ICA (tensor-PICA), has been proposed [12-14]. This method simultaneously decomposes group fMRI data into modes describing variations across space, time, and subjects. It has been demonstrated that the tensor-PICA approach can provide useful representations of group fMRI data in resting-state studies $[15,16]$.

Although the two methods each have their pros and cons [17], both of them are widely used in resting-state fMRI studies of neuropsychiatric disorders. Additionally, 
some findings using the two methods have yielded consistent results. But some inconsistent results have been reported. In the following section, we will comprehensively review the progress obtained by using each of the two methods from individual ROI analysis, to local network analysis, to whole brain network analysis (Table 1).

Table 1. Methods for investigating spatial patterns of SLFFs and progress in understanding neuropsychiatric disorders

\begin{tabular}{|c|c|c|c|}
\hline Authors & Disorders & Patient/Control & Methods \\
\hline Greicius et al (2004) & AD, mild & $14 / 14$ & ICA \\
\hline Wang L et al (2006) & $\mathrm{AD}$, early & $13 / 13$ & $\begin{array}{l}\text { Correlation analysis of } \\
\text { individual ROIs }\end{array}$ \\
\hline & & & $\begin{array}{l}\text { Correlation analysis of the } \\
\text { whole brain and of individual }\end{array}$ \\
\hline Wang K et al (2007) & AD, early & $14 / 14$ & ROIs \\
\hline Allen et al (2007) & AD, probable & $8 / 8$ & $\begin{array}{l}\text { Correlation analysis of } \\
\text { individual ROIs }\end{array}$ \\
\hline Sorg et al (2007) & aMCI & $24 / 16$ & $\begin{array}{l}\text { ICA, correlation analysis of } \\
\text { individual ROIs, structure }\end{array}$ \\
\hline Liang et al (2006b) & Schizophrenia & $15 / 15$ & $\begin{array}{l}\text { Correlation analysis of the } \\
\text { whole brain }\end{array}$ \\
\hline Bluhm et al (2007) & $\begin{array}{l}\text { Schizophrenia, } \\
\text { chronic and } \\
\text { medicated }\end{array}$ & $17 / 17$ & $\begin{array}{l}\text { Correlation analysis of } \\
\text { individual ROIs }\end{array}$ \\
\hline Garrity et al (2007) & Schizophrenia & $21 / 22$ & ICA \\
\hline Zhou et al (2007a) & $\begin{array}{l}\text { Schizophrenia, } \\
\text { first-episode }\end{array}$ & $17 / 17$ & $\begin{array}{l}\text { Correlation analysis of } \\
\text { individual ROIs }\end{array}$ \\
\hline Zhou et al (2007b) & $\begin{array}{l}\text { Schizophrenia, } \\
\text { paranoid }\end{array}$ & $18 / 18$ & $\begin{array}{l}\text { Correlation analysis of multiple } \\
\text { ROIs }\end{array}$ \\
\hline Calhoun et al (2008) & $\begin{array}{l}\text { Schizophrenia, } \\
\text { chronic }\end{array}$ & $20 / 20$ & ICA \\
\hline Zhou et al (2008) & $\begin{array}{l}\text { Schizophrenia, } \\
\text { paranoid }\end{array}$ & $17 / 14$ & $\begin{array}{l}\text { Correlation analysis } \\
\text { individual ROIs }\end{array}$ \\
\hline
\end{tabular}

\section{1 Individual ROI analysis and progress in understanding neuropsychiatric disorders:}

RsFC based on a single ROI is the most common method for investigating restingstate functionality in neuropsychiatric disorders. The key point of this method is to select an appropriate region as ROI. An ideal ROI should satisfy the following criteria: (1) implicated in a pathological lesion or cognitive dysfunction associated with the brain disorder to be investigated; (2) demonstrated as a local abnormality in anatomy or function in a previous study. Using rsFCs based on an individual ROI has led to some interesting findings for $\mathrm{AD}$ and schizophrenia as reviewed below. 
$A D$ : Two regions in the default mode network (DMN), the hippocampus and the posterior cingulate cortex (PCC), have attracted the most attention due to their roles in memory and their morphologic and metabolic abnormalities in AD patients [18]. As a core region in the DMN (this network is also called the task-negative network, TNN), the PCC showed decreased correlations with other regions in the same network and decreased anti-correlations with regions in the task-positive network (TPN), which is anti-correlated with the TNN, in early AD compared to healthy controls [19]. Two other recent studies [20,21] independently investigated the hippocampal rsFC pattern in AD. Both of the studies found that the hippocampus exhibited disrupted rsFCs with extensive regions, including the PCC/PCu. However, Wang and coworkers [21] also found increased rsFCs between the left hippocampus and the right lateral prefrontal cortex, which was absent in Allen's study [20]. The differences in results between the two studies likely arose from differences in the ROIs that were selected, from differences in disease severity [20] and from differences between the sample subjects (for a review, see [22]. In a recent study, healthy subjects exhibited differences in rsFC patterns between the anterior hippocampus and the posterior portion, including the body of the hippocampus and the posterior parahippocampus [23]. Subtle differences in anterior-posterior hippocampal rsFCs may account for the differences in the two studies, which selected different subregions of the hippocampus as ROIs.

Schizophrenia: The dorsolateral prefrontal cortex (DLPFC), because of its local abnormalities in anatomy and function [24] and its role in various neural circuits relevant to the anatomical and physiological mechanisms of cognitive dysfunction in schizophrenia, has attracted the attention of Zhou and colleagues [25]. By examining the FC pattern of DLPFC in patients with first-episode schizophrenia and matched controls, these researchers from our laboratory found that the DLPFC exhibited decreased rsFC with the PCC, among other regions [25]. Because the regions and functions of the default mode network have been linked with schizophrenia, the PCC, as a core region in this network, was selected as the ROI to compare the SLFF pattern of chronic, medicated schizophrenic patients with that of control subjects [26]. The PCC was found to show decreased rsFCs in regions associated with the DMN including the PCC, lateral parietal, medial prefrontal cortex (MPFC) and cerebellar regions in chronic, medicated schizophrenic patients [26]. Considering that the hippocampus has been implicated as participating in the pathophysiology of schizophrenia, the FC pattern of the anterior hippocampus was also investigated. In patients with schizophrenia, the bilateral hippocampi showed reduced rsFCs to some regions which have been reported to be involved in episodic memory, such as the PCC, the extrastriate cortex, the MPFC, and the parahippocampal gyrus, which may reflect the disconnectivity within a neural network related to the anterior hippocampus in schizophrenia [27].

Comments on this method: rsFC based on an individual ROI can easily be performed and can provide distinct information on specific regions implicated in neuropsychiatric disorders. However, limitations of this method need to be kept in mind. The method strongly depends on prior knowledge about the related disorders. In published studies, the ROI was selected according to the researchers' interest and outlined by hand [20, 21, 27] selected by software [19, 25] or identified by known coordinates [26, 28] This subjectivity in ROI selection has lead to slight differences in the anatomical location of the ROIs, which led to differences in the ROI FC patterns, 
as we noted in the case of the discrepancy in the hippocampal FC in AD [20, 21]. In addition, information outside the functional connectivity pattern of a specific ROI cannot be obtained by this method.

\subsection{Local network analyses and progress in understanding neuropsychiatric disorders:}

Brain circuits or networks have often been implicated in major neuropsychiatric disorders, such as AD and schizophrenia [29-31], thus the investigation of rsFC within a specific network may improve our understanding of the neural basis of these disorders. This is supported by findings that have revealed the presence of a number of resting-state brain networks, such as the DMN (e.g. TNN) [8, 32, 33], the TPN [32, 33], and the dorsal and ventral attention networks [34] in healthy subjects. Additionally, these networks are known to be related to emotion, memory, attention, and other high brain functions, which have often been observed to be impaired in neuropsychiatric disorders.

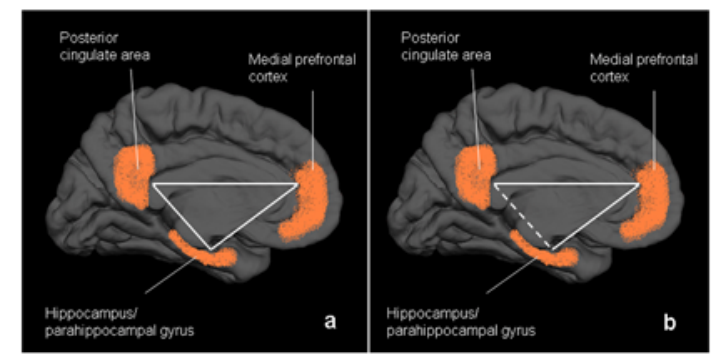

Fig. 1. Altered functional connectivities in the default mode network in Alzheimer's disease. a. The core brain regions in the default mode network in healthy subjects are illustrated schematically. Prominent components of this network include medial prefrontal regions, posterior regions in the medial and lateral parietal cortex, the lateral temporal cortex and the medial temporal lobe (including the hippocampus and parahippocampal gyrus). Regions within this core brain system are functionally correlated with each other and, prominently, with the hippocampal formation. The solid line represents the correlations between the core regions. $b$. The functional connectivity between the hippocampal formation and the medial posterior regions were consistently found to be decreased or absent in patients with Alzheimer's disease (for references, please see the main text). The dashed line represents decreased or absent correlations between the hippocampus/parahippocampal gyrus and the posterior cingulate area.

$A D$ : Using ICA to isolate the DMN during a simple sensory-motor task, Greicius et al (2004) found decreased connectivity within the DMN in an AD group [35], which is consistent with findings from rsFC based on individual ROIs [19-21]. By applying ICA and ROI-based FC analysis, Sorg et al. further found that the patients with amnestic mild cognitive impairment (aMCI), a syndrome that carries a high risk for developing $\mathrm{AD}$, demonstrated reduced network-related activity in selected areas of the DMN (left PCC and right MPFC) [36]. These studies consistently show that disconnection in the DMN is a distinctive characteristic of AD (Fig. 1). In addition to 
the DMN, Sorg et al. found the executive attention network to be affected, but the remaining resting networks to be intact, in individuals at high risk for $\mathrm{AD}$. The reduced network-related activity in the executive attention network is in line with observed attentional deficits in MCI and AD, indicating impaired interaction between the two anti-correlated networks (TPN and TNN) that prominently organize intrinsic brain activity [36].

Schizophrenia: Aberrant FC within the DMN has been observed in schizophrenia using ICA [37], demonstrating increased activity in the ACC/MPFC, the parahippocampus and the PCC in patients. These researchers also found that patients with schizophrenia showed significantly more high-frequency fluctuations and controls showed significantly more low-frequency fluctuations in the DMN [37]. This pattern was validated and extended to the rest of the resting-state networks in a later study [38]. By directly investigating the interregional rsFCs among the regions constituting the DMN, increased rsFCs within this network were also observed in schizophrenia [39]. The FCs, that primarily increased within the TPN, as well as increased anti-correlations between the two networks were also found in this disease [39]. These findings suggest that these abnormalities related to the DMN could be a possible source for the abnormalities in information processing activities that are characteristic of patients with schizophrenia.

In addition to the TPN and TNN, the frontostriatal system has also attracted the attention of researchers. Salvador et al. used the mutual information measure to compare the resting-state connections among three main components of the frontostriatal system (DLPFC, the basal ganglia, and the thalami) in schizophrenia and in healthy controls. Increased connectivity between the DLPFC and the basal ganglia in schizophrenia were consistently found across low, medium and high frequency bands [40]. These increased connectivities could potentially be responsible for dysfunctions in the frontostriatal loop in patients with schizophrenia.

Comments on this method: Identification of the affected regions is the primary prerequisite for investigations into the rsFC within a disease-related network. However, determining which regions should be recruited for this purpose is limited by the prior knowledge of the researchers. Although some methods, such as ICA, can greatly reduce the researcher's subjectivity, some key steps, such as deciding on the number of components and determining how to classify each component into noise or physiologically meaningful signals, still depends on the subjective opinions of researchers. This subjectivity leads to differences in the regions that are determined to constitute a network and thus leads to difficulty in comparing results across studies. Furthermore, the functionality and physiological meaning of these resting-state networks need to be further clarified. With this in mind, caution must be exercised in interpreting the findings obtained by comparing the rsFC in the resting-state networks of patients with that of controls.

\subsection{Whole brain network analyses and progress in understanding neuropsychiatric disorders:}

In contrast to local network-based interregional FC, which focuses on the FCs associated with a few preselected seed regions within a specific network or circuit 
while ignoring other potentially interesting patterns of connectivity, whole brain network-based FC analysis can objectively and comprehensively detect altered FCs throughout an entire brain level by automatically dividing the entire brain into multiple regions and performing correlation or partial correlation analysis on each pair of these regions. This method was first used in a single patient who was minimally conscious following a brainstem lesion [41] and then was developed and used in AD and schizophrenia [19, 42] (Fig. 2).
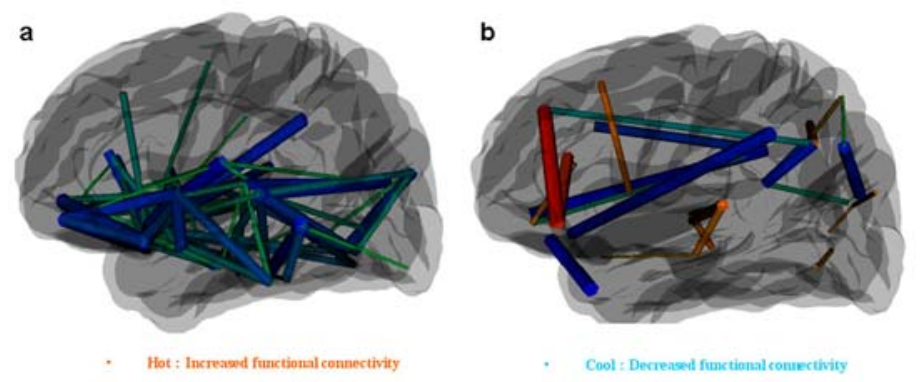

Fig. 2. Altered resting-state functional connectivity in schizophrenia and Alzheimer's disease. a. Schizophrenia patients mainly showed decreased functional connectivities and such abnormalities were widely distributed throughout the entire brain rather than restricted to a few specific brain regions. b. Alzheimer's disease mainly showed decreased functional connectivities between the prefrontal and parietal lobes, but increased functional connectivities within the prefrontal lobe, parietal lobe and occipital lobe.

$A D$ : Wang et al. (2007) found that AD patients show many decreased rsFCs, which are mainly between the prefrontal and parietal lobes, but these patients also show increased rsFCs mainly between regions within lobes, such as within the prefrontal lobe, within the parietal lobe, or within the occipital lobe [19]. These findings are compatible with the anterior-posterior disconnection phenomenon and compensatory effect within lobes observed previously in AD patients (for review, see [22]. More interestingly, by using whole brain network analysis, the authors also found decreased anti-correlations between the two abovementioned intrinsically anti-correlated networks (TPN and TNN), again suggesting that disturbance of the balance between the intrinsically anti-correlated networks may be associated with attention deficits in AD patients.

Schizophrenia: Liang et al. (2006) found that in patients with schizophrenia the decreased FCs were widely distributed throughout the entire brain, although most of them were related to the insula, the temporal lobe (including the medial temporal structures), the prefrontal lobe and the corpus striatum. Increased FCs were mainly related to the cerebellum in patients. Although in this preliminary study, interregional anti-correlation was not considered, which made it difficult to directly compare the distribution of these altered FCs with that in other disorders, this study provides further support for the hypothesis that schizophrenia may arise from the disrupted functional integration of widespread brain areas [42]. 
Comments on this method: By comparing the global distribution of these altered FCs in different disorders, it is possible to find various disease-related characteristics and thus to differentiate different disorders. However, some issues need to be addressed. First, the findings obtained by this method are affected by anatomical parcellation. Therefore, it is necessary to pay attention to the fact that mapping the resulting whole brain network using different templates may induce different findings [43]. Secondly, current automatic registration techniques make it difficult to guarantee the exact match of some small gyri/sulci, especially in the cerebellar lobes, across subjects. Finally, inter-subject variability in the anatomical regions must also be considered carefully in future studies.

\section{Conclusions}

By analyzing the spatial pattern of spontaneous BOLD activity, some disease-related abnormalities can be obtained. Disrupted rsFCs within the DMN, especially those associated with the hippocampus and the PCC, may be a distinctive characteristic of $\mathrm{AD}$ (Fig. 1). The regions showing disrupted rsFCs are highly similar to those that show pathology in the early stages of the disease, as measured by molecular imaging of amyloid plaques using PET, and those that are affected by structural atrophy, as measured by longitudinal MRI [18]. Decreased rsFCs within the attention relatednetworks is another consistent finding in $\mathrm{AD}$ and is consistent with observed attention deficits in MCI and AD. In schizophrenia, aberrant rsFCs within the DMN are found, but the main difference is that the strength of the rsFCs are abnormally increased in patients. By analyzing the clinical correlates of the strength of the rsFC, the rsFCs associated with the component regions of the DMN are found to vary with positive symptoms measured by different clinical scales [26, 27, 37]. The positive symptomdependent correlation of the component region in the DMN suggests that functional dysconnectivity in the DMN may be a reflection of an impaired self-monitoring function in schizophrenia, which could lead to positive symptoms such as hallucinations and delusions [44].

In conclusion, altered activity patterns of SLFFs have been found in the major neuropsychiatric disorders, whether at a local level or a global level. These studies highlight the usefulness of resting-state fMRI for studying the brain in neuropsychiatric disorders. More importantly, these studies suggest the possibility that the altered SLFFs in neuropsychiatric disorders could be valuable brain imaging biomarkers for diagnosis, therapy evaluation and prognosis.

Acknowledgments. The authors thank Drs. Rhoda E. and Edmund F. Perozzi for English language assistance. This work was supported by the Natural Science Foundation of China (Grant No. 30425004 and Grant No. 30730035), and the National Key Basic Research and Development Program (973) (Grant No. 2007CB512304) 


\section{References}

1. Fox, M.D. and Raichle, M.E., Spontaneous fluctuations in brain activity observed with functional magnetic resonance imaging. Nat. Rev. Neurosci. 8 (2007) 700-11.

2. Bandettini, P.A. and Bullmore, E., Endogenous oscillations and networks in functional magnetic resonance imaging. Hum. Brain. Mapp. 29 (2008) 737-9.

3. Kiviniemi, V., Endogenous brain fluctuations and diagnostic imaging. Hum. Brain. Mapp. 29 (2008) 810-817.

4. Zeki, S. and Shipp, S., The functional logic of cortical connections. Nature, 335 (1988) 3117.

5. Friston, K.J., Functional and effective connectivity in neuroimaging: A synthesis. Hum Brain Mapp, 2 (1994) 56-78.

6. Friston, K.J., Frith, C.D., Liddle, P.F., and Frackowiak, R.S., Functional connectivity: the principal-component analysis of large (PET) data sets. J. Cereb. Blood. Flow. Metab. 13 (1993) 5-14.

7. Calhoun, V.D., Adali, T., Pearlson, G.D., and Pekar, J.J., A method for making group inferences from functional MRI data using independent component analysis. Hum Brain Mapp, 14 (2001) 140-51.

8. Greicius, M.D., Krasnow, B., Reiss, A.L., and Menon, V., Functional connectivity in the resting brain: a network analysis of the default mode hypothesis. Proc. Natl. Acad. Sci. U S A. 100 (2003) 253-8.

9. Bartels, A. and Zeki, S., The chronoarchitecture of the cerebral cortex. Philos. Trans. R. Soc. Lond. B. Biol. Sci. 360 (2005) 733-50.

10.van de Ven, V.G., Formisano, E., Prvulovic, D., Roeder, C.H., and Linden, D.E., Functional connectivity as revealed by spatial independent component analysis of fMRI measurements during rest. Hum Brain Mapp, 22 (2004) 165-78.

11.Kiviniemi, V., Kantola, J.H., Jauhiainen, J., Hyvarinen, A., and Tervonen, O., Independent component analysis of nondeterministic fMRI signal sources. Neuroimage. 19 (2003) 25360 .

12.Beckmann, C.F., DeLuca, M., Devlin, J.T., and Smith, S.M., Investigations into resting-state connectivity using independent component analysis. Philos. Trans. R. Soc. Lond. B. Biol. Sci. 360 (2005) 1001-13.

13.Beckmann, C.F. and Smith, S.M., Tensorial extensions of independent component analysis for multisubject FMRI analysis. Neuroimage. 25 (2005) 294-311.

14.De Luca, M., Beckmann, C.F., De Stefano, N., Matthews, P.M., and Smith, S.M., fMRI resting state networks define distinct modes of long-distance interactions in the human brain. Neuroimage. 29 (2006) 1359-67.

15.Damoiseaux, J.S., Rombouts, S.A., Barkhof, F., Scheltens, P., Stam, C.J., Smith, S.M., and Beckmann, C.F., Consistent resting-state networks across healthy subjects. Proc. Natl. Acad. Sci. U S A. 103 (2006) 13848-53.

16.Fransson, P., Skiold, B., Horsch, S., Nordell, A., Blennow, M., Lagercrantz, H., and Aden, U., Resting-state networks in the infant brain. Proc. Natl. Acad. Sci. U S A. 104 (2007) 15531-6.

17.Fox, M.D., Snyder, A.Z., Vincent, J.L., and Raichle, M.E., Intrinsic Fluctuations within Cortical Systems Account for Intertrial Variability in Human Behavior. Neuron. 56 (2007) 171-184.

18.Buckner, R.L., Andrews-Hanna, J.R., and Schacter, D.L., The brain's default network: anatomy, function, and relevance to disease. Ann. N. Y. Acad. Sci. 1124 (2008) 1-38.

19.Wang, K., Liang, M., Wang, L., Tian, L., Zhang, X., Li, K., and Jiang, T., Altered functional connectivity in early Alzheimer's disease: A resting-state fMRI study. Hum Brain Mapp, 28 (2007) 967-978. 
20.Allen, G., Barnard, H., McColl, R., Hester, A.L., Fields, J.A., Weiner, M.F., Ringe, W.K., Lipton, A.M., Brooker, M., McDonald, E., Rubin, C.D., and Cullum, C.M., Reduced hippocampal functional connectivity in Alzheimer disease. Arch Neurol, 64 (2007) 1482-7.

21.Wang, L., Zang, Y., He, Y., Liang, M., Zhang, X., Tian, L., Wu, T., Jiang, T., and Li, K., Changes in hippocampal connectivity in the early stages of Alzheimer's disease: evidence from resting state fMRI. Neuroimage. 31 (2006) 496-504.

22.Liu, Y., Wang, K., Yu, C., He, Y., Zhou, Y., Liang, M., Wang, L., and Jiang, T., Regional homogeneity, functional connectivity and imaging markers of Alzheimer's disease: A review of resting-state fMRI studies. Neuropsychologia, (2008).

23.Kahn, I., Andrews-Hanna, J.R., Vincent, J.L., Snyder, A.Z., and Buckner, R.L., Distinct Cortical Anatomy Linked to Subregions of the Medial Temporal Lobe Revealed by Intrinsic Functional Connectivity. J Neurophysiol, (2008).

24.Bunney, W.E. and Bunney, B.G., Evidence for a compromised dorsolateral prefrontal cortical parallel circuit in schizophrenia. Brain Res Brain Res Rev, 31 (2000) 138-46.

25.Zhou, Y., Liang, M., Jiang, T., Tian, L., Liu, Y., Liu, Z., Liu, H., and Kuang, F., Functional dysconnectivity of the dorsolateral prefrontal cortex in first-episode schizophrenia using resting-state fMRI. Neurosci Lett, 417 (2007) 297-302.

26.Bluhm, R.L., Miller, J., Lanius, R.A., Osuch, E.A., Boksman, K., Neufeld, R.W., Theberge, J., Schaefer, B., and Williamson, P., Spontaneous low-frequency fluctuations in the BOLD signal in schizophrenic patients: anomalies in the default network. Schizophr. Bull. 33 (2007) 1004-12.

27.Zhou, Y., Shu, N., Liu, Y., Song, M., Hao, Y., Liu, H., Yu, C., Liu, Z., and Jiang, T., Altered resting-state functional connectivity and anatomical connectivity of hippocampus in schizophrenia. Schizophr. Res. 100 (2008) 120-132.

28.Castellanos, F.X., Margulies, D.S., Kelly, C., Uddin, L.Q., Ghaffari, M., Kirsch, A., Shaw, D., Shehzad, Z., Di Martino, A., Biswal, B., Sonuga-Barke, E.J., Rotrosen, J., Adler, L.A., and Milham, M.P., Cingulate-precuneus interactions: a new locus of dysfunction in adult attention-deficit/hyperactivity disorder. Biol Psychiatry, 63 (2008) 332-7.

29.Delbeuck, X., Van der Linden, M., and Collette, F., Alzheimer's disease as a disconnection syndrome? Neuropsychol Rev, 13 (2003) 79-92.

30.Friston, K.J. and Frith, C.D., Schizophrenia: a disconnection syndrome? Clin Neurosci, 3 (1995) 89-97.

31.Friston, K.J., The disconnection hypothesis. Schizophr. Res. 30 (1998) 115-25.

32.Fransson, P., Spontaneous low-frequency BOLD signal fluctuations: an fMRI investigation of the resting-state default mode of brain function hypothesis. Hum Brain Mapp, 26 (2005) $15-29$

33.Fox, M.D., Snyder, A.Z., Vincent, J.L., Corbetta, M., Van Essen, D.C., and Raichle, M.E., The human brain is intrinsically organized into dynamic, anticorrelated functional networks. Proc. Natl. Acad. Sci. U S A. 102 (2005) 9673-8.

34.Fox, M.D., Corbetta, M., Snyder, A.Z., Vincent, J.L., and Raichle, M.E., Spontaneous neuronal activity distinguishes human dorsal and ventral attention systems. Proc. Natl. Acad. Sci. U S A. 103 (2006) 10046-51.

35.Greicius, M.D., Srivastava, G., Reiss, A.L., and Menon, V., Default-mode network activity distinguishes Alzheimer's disease from healthy aging: evidence from functional MRI. Proc. Natl. Acad. Sci. U S A. 101 (2004) 4637-42.

36.Sorg, C., Riedl, V., Muhlau, M., Calhoun, V.D., Eichele, T., Laer, L., Drzezga, A., Forstl, H., Kurz, A., Zimmer, C., and Wohlschlager, A.M., Selective changes of resting-state networks in individuals at risk for Alzheimer's disease. Proc. Natl. Acad. Sci. U S A. 104 (2007) 18760-5.

37.Garrity, A.G., Pearlson, G.D., McKiernan, K., Lloyd, D., Kiehl, K.A., and Calhoun, V.D., Aberrant "default mode" functional connectivity in schizophrenia. Am. J. Psychiatry. 164 (2007) 450-7. 
38.Calhoun, V.D., Kiehl, K.A., and Pearlson, G.D., Modulation of temporally coherent brain networks estimated using ICA at rest and during cognitive tasks. Hum Brain Mapp. 29 (2008) 828-38.

39.Zhou, Y., Liang, M., Tian, L., Wang, K., Hao, Y., Liu, H., Liu, Z., and Jiang, T., Functional disintegration in paranoid schizophrenia using resting-state fMRI. Schizophr. Res. 97 (2007) 194-205.

40.Salvador, R., Martinez, A., Pomarol-Clotet, E., Sarro, S., Suckling, J., and Bullmore, E., Frequency based mutual information measures between clusters of brain regions in functional magnetic resonance imaging. Neuroimage. 35 (2007) 83-8.

41.Salvador, R., Suckling, J., Coleman, M.R., Pickard, J.D., Menon, D., and Bullmore, E., Neurophysiological architecture of functional magnetic resonance images of human brain. Cereb. Cortex. 15 (2005) 1332-42.

42.Liang, M., Zhou, Y., Jiang, T., Liu, Z., Tian, L., Liu, H., and Hao, Y., Widespread functional disconnectivity in schizophrenia with resting-state functional magnetic resonance imaging. Neuroreport. 17 (2006) 209-13.

43.Wang, J., Wang, L., Zang, Y., Yang, H., Tang, H., Gong, Q., Chen, Z., Zhu, C., and He, Y., Parcellation-dependent small-world brain functional networks: A resting-state fMRI study. Hum Brain Mapp, 30 (2009) 1511-1523.

44.Williamson, P., Are anticorrelated networks in the brain relevant to schizophrenia? Schizophr. Bull. 33 (2007) 994-1003. 